\title{
Characterization of Main-Chain Liquid Crystal Elastomers Using X-ray Diffraction Method
}

\author{
Supardi $^{1,2, a}$, Yusril Yusuf ${ }^{2}$, and Harsoyo ${ }^{2, b}$ \\ ${ }^{1}$ Physics Education Department, Yogyakarta State University, \\ Karangmalang, Yogyakarta Indonesia \\ ${ }^{2}$ Physics Department, Gadjah Mada University, Bulaksumur, \\ Yogyakarta, Indonesia \\ asupardi@uny.ac.id, busril@ugm.ac.id
}

\begin{abstract}
We characterized the four main chain liquid crystal elastomers (MCLCEs) samples using the $x$-ray diffraction method. The four samples had different concentration of crosslinkers, i.e $8 \%, 12 \%, 14 \%$ and $16 \%$. There was a relationship between crosslinkers concentration and d-spacing parameter here, namely when the concentration increased the d-spacing parameter did, too. In polymeric material, the dspacing parameter indicates the average of length between dominant clusters of the heavy molecule. We also calculated the degree of crystallinity and amorphousinity based on the difractogram obtained from the characterization and associated them to their mechanical properties. We found that as the degree of crystallinity decreased (or degree of amorphousinity increased) the sample will be more elastic, and vice versa.
\end{abstract}

Keywords- MCLCEs, crosslinkers concentration, crystallinity degrees.

\section{INTRODUCTION}

Liquid Crystal Elastomers (LCEs), both MCLCEs and SCLCEs (Side Chain Liquid Crystal Elastomers) are polymers produced by crosslinking of liquid crystal polymers covalently with monomers are mesogen units forming a three dimension network [1,2,3]. Mechanical properties of the LCEs can be controlled by selection of liquid crystal phase, density of crosslinkers, their flexible polymer backbones, merger of the backbones, the group of liquid crystals and external stimuli $[1,2,4]$. The MCLCE materials were synthesized by the reaction of vinyl-or vinoloxy-end mesogens under condition hydrosilylation with crosslinker agent was pentamethylcyclopentasiloxane $\left(\mathrm{C}_{5} \mathrm{H}_{20} \mathrm{O}_{5} \mathrm{Si}_{2}\right)$ and the mesogen unit was monomer 2-ethyl1,4-phenylen bis [4 - [4 - (viniloxy) buboxy] benzoate] $\left(\mathrm{C}_{34} \mathrm{H}_{38} \mathrm{O}_{6}\right)$, chain extender 1,1,3,3,-tetramethyldisiloxane $\left(\mathrm{C}_{4} \mathrm{H}_{14} \mathrm{O} \mathrm{Si}_{2}\right)$ [4,5]. Some studies conducted by previous researcher to show that the MCLCEs have more promising properties compared SCLCEs because of its response to external stimuli such as heat and magnetic field [6].

As a polymer material, MCLCEs can be in a state that is a mixture of crystalline and amorphous. Crystalline state is shown by the regularity of mesogen units in the polymer crosslinking in directions parallel to the director $\mathbf{n}$, while the amorphous state is indicated by the irregularity of mesogens directions. The two states make the polymeric materials generally have a strong properties. Specially for MCLCE materials, crosslinker agents were added to crosslink between the liquid crystal polymers. Consequently, The MCLCEs have not only strong but elastic properties.

In previous study, we examined the mechanical properties of these materials when given external stimuli in the form of heat. The results showed that the concentration of crosslinker agents was very influential on the optimum value of expansion and contraction where there was a linear relationship between crosslinker concentrations and their optimum value [2]. This result was consistent with the assumption that the addition of crosslinker concentration will loosen the bonds in the liquid crystal polymers so its more elastic.

In this paper, we present the results of study of the microscopic structure of the MCLCE materials using x-ray diffraction method. We examined four MCLCE samples with different concentration of crosslinkers. We conducted this experiment at room temperature, so that the liquid crystals in the MCLCE samples entirely are in the nematic state. We try to explain why there was a change in the dspacing parameter where the parameter indicates the average of distances between dominant clusters of the heavy molecular. We also calculated the degree of crystallinity through their diffractogram by comparing the area of crystalline or amorphous state to the sum of crystalline and amorphous area.

\section{EXPERIMENTAL}

$\mathrm{X}$-ray diffraction is a method that is widely used to investigate the crystal structure in the solid by looking at the peaks of strong scattering. The crystal structure will provide a strong scattering when the direction of the crystal planes forms an angle to the x-ray beam and satisfies the Bragg equation [7],

$$
2 d \sin \theta=n \lambda
$$

where $d$ : distance between crystal planes, $\theta$ : angle of deviation, $n$ : orde (integer number: $0,1,2,3, \ldots$ ), and $\lambda$ : wavelength. For polymeric materials, the x-ray diffraction method provide information about the structure of the polymer shown by the crystalline and amorphous state mixing randomly. The patterns of x-ray scattering in 
polymers give an approximate information about the degree of crystallinity by comparing the area of crystalline state to the total of the crystalline and amorphous area.

In this research, we set up four MCLCE samples with crosslinker concentration of $8 \%, 12 \%, 14 \%$, and $16 \%$ where each sample has a glass transition $-1^{\circ} \mathrm{C}, 8^{\circ} \mathrm{C}, 6^{\circ} \mathrm{C}$, and $8^{\circ} \mathrm{C}$ respectively. We prepare these materials with dimension size of $\sim 1.0 \mathrm{~cm} \times 0.8 \mathrm{~cm}$. We characterize the four samples by using Miniflex Rigaku x-ray diffractometer $600 \mathrm{~W}$ generator with copper target and the $2 \theta$ scan range of $2^{\circ}$ to $145^{\circ}$.

We measured the 4 samples with $2 \theta$ range from $2^{\circ}$ to $90^{\circ}$. It takes time for 15 minutes to obtain every data for each sample. By using the software installed to the computer that is connected directly to the x-ray diffractometer, we obtained some data in the text format. We then used WinPlotr software to create a difractogram displaying the relationship between intensity in counts per second (cps) and parameter $2 \theta$ in degree. Through the diffractogram, we analyzed and determined the degree of crystallinity for each sample.

\section{RESULTS AND DISCUSSION}

MCLCEs have strong and elastic properties since they have a mixed state, namely crystalline and amorphous state. The crystalline state causes them to have an ability to retain its original form, while the amorphous state causes the materials flexible and has a memory to return to its original shape. The liquid crystal polymers in the form of mesogen units in MCLCEs make this material very special compared to the common polymers, because of its existence the polymer can be controlled by external stimuli such as heat. These properties make the materials to have a potential candidate as an artificial muscle in the future.

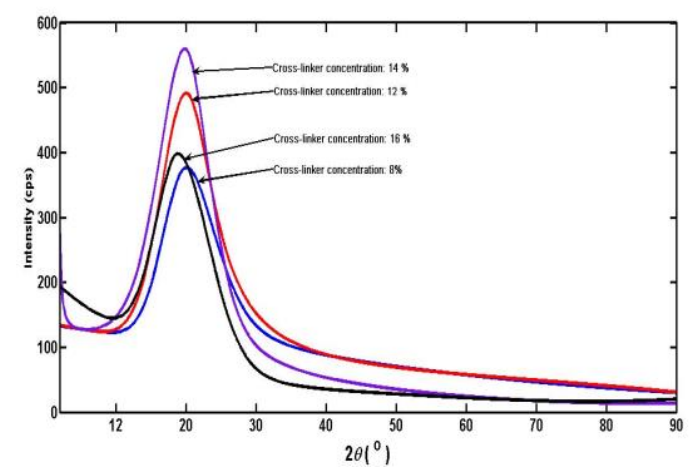

Figure 1. Diffractogram of MCLCE samples using x-ray diffraction method for $8 \%, 12 \%, 14 \%$, and (d) $16 \%$ of cross-linker concentrations.

We characterized the MCLCE samples using x-ray diffraction method. Fig. 1 shows the test results for the four MCLCE samples with concentration of crosslinkers are $8 \%$, $12 \%, 14 \%$ and $16 \%$. The presence of the amorphous state in the samples lead to the broadening of their peaks. We noticed that none of the four samples show a sharp peak as in crystalline materials. Obviously, there is a mixture between a crystalline and amorphous state with different degree of crystallinity depending on the crosslinker concentration given in this elastomer.

Table 1. X-ray diffraction parameters of MCLCE sample.

\begin{tabular}{|c|c|c|c|c|c|}
\hline No & $\begin{array}{c}\text { Crosslinkers } \\
\text { concentration }\end{array}$ & $\begin{array}{c}2 \theta \\
(\text { degree })\end{array}$ & $\begin{array}{c}d \\
\left(\mathrm{x} 10^{-10}\right. \\
\text { meter })\end{array}$ & $\begin{array}{c}\text { Height } \\
(\mathrm{cps})\end{array}$ & $\begin{array}{c}\text { FWHM } \\
(\text { degree })\end{array}$ \\
\hline 1 & $8 \%$ & 20.13 & 4.408 & $\begin{array}{c}2265 \\
(168)\end{array}$ & 9.07 \\
\hline 2 & $12 \%$ & 20.10 & 4.414 & $\begin{array}{c}3328 \\
(204)\end{array}$ & 9.01 \\
\hline 3 & $14 \%$ & 19.89 & 4.460 & $\begin{array}{c}4073 \\
(226)\end{array}$ & 8.94 \\
\hline 4 & $16 \%$ & 18.97 & 4.467 & $\begin{array}{c}2725 \\
(185)\end{array}$ & 9.27 \\
\hline
\end{tabular}

Table 1 presents the quantitative results of these experiments for the measured quantities, i.e $2 \theta$ : deviation angle, d-spacing: the average of distances between dominant clusters of the heavy atoms (d-spacing), and the full width half maximum (FWHM). It shows that there is a shift of the intensity peak and an increase in the $d$-spacing when crosslinker concentration of the samples is raised, i.e 0.4408 $\mathrm{nm}$ for the sample with crosslinker concentration $8 \%$, $0.4414 \mathrm{~nm}$ for $12 \%, 0.4460 \mathrm{~nm}$ for $14 \%$, and $0.4467 \mathrm{~nm}$ for $16 \%$ (see Fig.2). This increase indicates that the intercalation was occurred between clusters of heavy atoms ( $\mathrm{Si}$ ) in liquid crystals polymer with the heavy atoms in the crosslinker molecules. When the concentration of crosslinker is increased from $8 \%$ to $12 \%$, the average distance between clusters of heavy atoms grows gradually. The increase of the average distance occurs drastically when the concentration of crosslinkers is increased from $14 \%$ to $16 \%$. Meanwhile, when the concentration is increased from $14 \%$ to $16 \%$, there is a tendency to move down gradually. This may be explained that the region between the crosslinker concentration $8 \%$ to $14 \%$, the crosslinker molecules still easily fit between the liquid crystals molecules. In contrast, in the region of concentration $14 \%$ to $16 \%$, the liquid crystal polymer molecules have already saturated to react with crosslinker molecules that $d$-spacing tends toward down.

Degree of Crystallinity. The degree of crystallinity of the polymer material is defined as the ratio of the crystalline area and a combined area of the crystalline and amorphous state given by [8]

$$
\chi_{c}=\frac{A_{\text {crystalline }}}{A_{\text {crystalline }}+A_{\text {amorph }}} \times 100 \%,
$$

where $A_{\text {crystalline }}$ is the area of crystalline state and $A_{\text {amorph }}$ is the area of amorphous state. 
Fig. 3 shows the results of calculation of the degree of crystallinity of each sample by using Eq. (2), and the results was fitted to the second order polynomial.

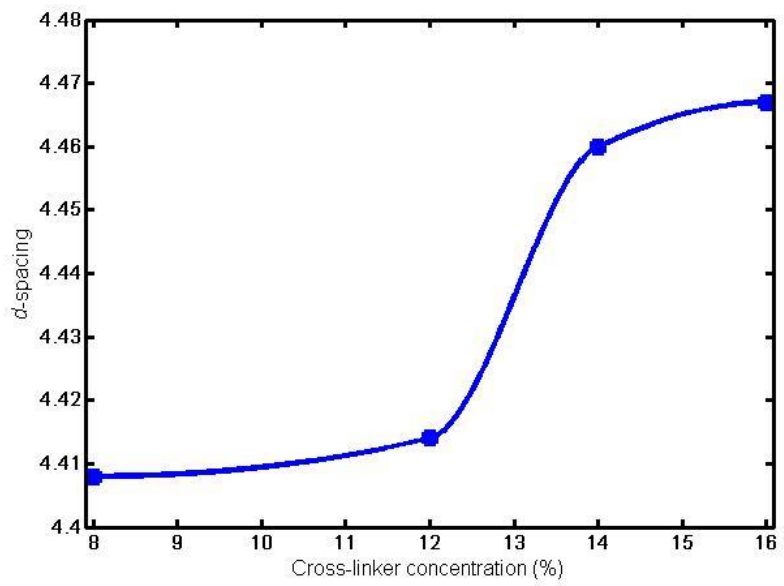

Figure 2. The relationship between $d$-spacing parameter and crosslinker concentrations. An increase of $d$-spacing indicates that an intercalation occured when crosslinker concentration was added to the sample.

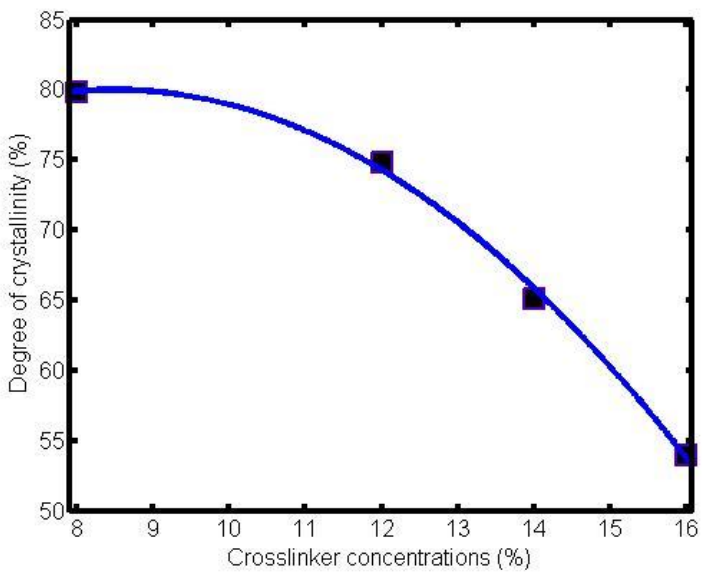

Figure 3. The relationship between degree of crystallinity and crosslinker concentrations. Blue solid lines show the fitting result using polynomial function.

The graph demonstrates that the change of crosslinker concentrations affects the degree of crystallinity, where the addition of crosslinker concentration causes it move down. The irregularity of molecules in the elastomer will decrease automatically the degree of crystallinity. As we know that by adding the crosslinker concentration in the MCLCE samples will increase the complexity of polymer chain due to the new branches were formed.

Referring to the previous studies that the maximum value of contraction and expansion of the MCLCE samples with crosslinker concentration 8\%,12\%,14\%, and 16\% were $\lambda_{x, \max }=70 \%, 87 \%, 96 \%, 108 \%$ and $\lambda_{y, \max }=$
$26 \%, 29 \%, 32 \%, 33 \%$ where $\lambda_{x, \max }$ is the maximum contraction and $\lambda_{y \text { max }}$ is the maximum expansion [2]. It indicates that elasticity of the samples were strongly influenced by concentration of crosslinker, where the greater the concentration of crosslinker, the greater the maximum contaction and expansion. In other words, if the elasticity is associated with the degree of crystallinity: the lower the degree of crystallinity the higher the elasticity of MCLCE samples.

\section{CONCLUSION}

We have studied four MCLCE samples with different concentrations of crosslinkers to determine their microscopic structure by using $\mathrm{x}$-ray diffraction method. We also calculated the degree of crystallinity of each sample to know the relationship between their microscopic and macroscopic properties. We concluded that (1) there was an increase of d-spacing parameter when the crosslinker concentrations increased. It was due to the intercalation of crosslinker molecules to the polymer liquid crystals, (2) the degree of crystallinity was strongly influenced by differences in the concentration of crosslinkers, where the higher the concentration of crosslinker the lower the degree of crystallinity. This was due to the increase in crosslinker concentration will increase the complexity of bonding in the material. (3) The elasticity of the MCLCE samples was closely related to the degree of crystallinity.

\section{REFERENCES}

[1] P. Xie and R. Zhang,"Liquid crystal elastomers, networks, and gels: advanced smart materials", J. Mater. Chem., vol. 15, pp. 2529-2550, February 2005.

[2] S. Hashimoto, Y. Yusuf, S. Krause, H. Finkelman, P. E. Cladis, H. R Brand, and S. Kai,"Multifunctional liquid crystal elastomers: Large electromechanical and electro-optical effects", App. Phys. Lett., vol. 92 , pp. 181902, May 2008.

[3] Dong-Uk Cho, Y. Yusuf, P.E Cladis, H.R Brand, H. Finkelman, and S. Kai," Trifunctionally Cross-Linked Liquid Single Crystal Elastomers: Swelling Dynamics and Electromechanical Effects",Jpn. J. App. Phys., Vol.46, pp. 1106-1113, March 2007.

[4] Supardi, Harsoyo and Y. Yusuf, "Experimental Studies of ThermoInduced Mechanical Effects in the Main-Chain Liquid Crystal Elastomers", Adv. Mat. Res., vol. 896, pp. 322-326, February 2014.

[5] Krause S., Zander F., Bergmann G., Brandt H., Wertmer H., and Finkelmann H., "Nematic mani-chain elastomers: Coupling and orientational behavior". C.R. Chimie. Vol. 12, pp. 85-104, February 2009.

[6] Y.Yusuf, P.E Cladis, H.R Brand, H. Finkelman, S. Kai, "Hystereses of volume changes in liquid single crystal elastomers swollen with low molecular weight liquid crystal", Chem. Phys. Lett., vol. 389, pp. 443-448, April 2004.

[7] David I. Bower, An Introduction to Polymer Physics, New York: Cambridge University Press, 2002, pp. 103-119.

[8] Ulf W. Gedde, Polymer Physics, London: Chapman \& Hall, 1995, pp 131-167. 